\title{
BROADER ONTOLOGICAL REFLECTIONS
}

When considering set theories as ontologies broader ontological issues arise.

On the one hand we have to reflect on ontological methodology. Maybe there are some methodological peculiarities to set theory as ontology. On the other hand we have to face some ontological issues that seem to arise especially with set theory. Foremost this is the issue of an incomplete universe. Also the distinction between sets and other collections has to be considered.

Famously Cantor distinguished sets as consistent collections or 'finished sets' from collections which cannot - on pains of antinomies - be taken as 'finished'. For these 'absolute infinite' collections (or 'multiplicities') Cantor claimed that 'the totality of their elements cannot be thought as "existing together" (cf. Cantor 1991, p.390). So they seem to have elements and at the same time the elements do not exist together. This looks like a contradiction - contradicting inter alia the Domain Principle and thus the name "inconsistent multiplicities" seems appropriate.

Another interesting issue in axiomatic ontology is the existence of a null set $\varnothing$. Zermelo at least sometimes thought of the null set as a mere technical device. Such an attitude does not square with set theoretic realism. $\varnothing$ is the paradigm abstract object. One may be tempted to locate the singleton of the Cologne Cathedral at Cologne, but there is nowhere to place $\varnothing$. One may try to avoid using $\varnothing$ and demand that every set has a member. Doing so (e.g. Maher 1968) requires to axiomatically introducing some substitute which behaves like $\varnothing$ (e.g. in building unions and cuts) without being $\varnothing$ (i.e. without being empty the substitute's members do not occur in the unions it builds). This way of employing axiomatic ontology to get rid of $\varnothing$ invites many questions for explanations, all of which do not arise in $\mathbf{Z}$ with respect to $\varnothing-$ or can be answered easily. Once one assumes an abstract category of containers it may not look so unnatural to assume $\varnothing$. The idea of an empty collection may be more natural than that of a sequence of transfinite cardinals. 
There are problems with a universal set even if the set theory containing it is consistent.

Although one may argue that a universal set is one of the hallmarks of an acceptable set theory, looking at the set theories with a universal set may give one second thoughts.

Set theories with a universal set attack the $2^{\text {nd }}$ objective to be achieved by a naïve set theory: having a universal set. The $1^{\text {st }}$ objective being having Naïve Comprehension. They can be taken as the claim that a universal set can be had without dialetheism. The more general reply to the dialetheist's claim (that we accept true contradictions because we want to see the two objectives of naïve set theory realized) might be that one sees more problems than benefits related to a universal set in the first place.

The first category of objections may be called negative in as much as they argue against the supposed gains of a universal set:

(i) Since meaning isn't extensional, fixing the meaning of "set" does not require an extension to the predicate "is a set". The meaning of "is a set" is fixed by the axioms governing our identification of sets.

(ii) If the extension of a predicate $\varphi$ cannot be a set this doesn't mean that there is no referent of $\varphi$ at all, it just has to be fixed singularly.

(iii) If consequence is spelled out model theoretically one may quantify over all appropriate structures' equivalence classes (in terms of isomorphy). Interpretations interpret languages with countably many formulas. Even sentences talking about uncountable structures cannot be supplied indefinitely. For structures of high enough ranks, even if they do not comprise all the sets, it is not obvious that some relevant structure is left out (this would have to be one with no isomorphic structures so far). A finite being cannot scrutinize them anyway.

The second category of objections may be called positive as they argue directly against a universal set as violating our intuitions itself:

(iv) As the absence of $U$ seems to miss having a set corresponding to sethood, $\mathbf{N F}$ and relatives have no set $[\epsilon],\{<\mathrm{x}, \mathrm{y}>\mid \mathrm{x} \in \mathrm{y}\}$, where $[\epsilon]$ is as intuitive as $U$ is, no singleton function etc.

(v) Like " $\in$ " " $\subseteq$ " could be taken as the basic concept of set theory. Then the powerset axiom is even more fundamental than it is intuitive already. $\wp(\mathrm{U}) \subseteq \mathrm{U}$ is not only a claim about cardinalities, but claims that all subsets of $U$ are already in $U$. This is counterintuitive, and to block Cantor's Theorem some subsets which seem to exist have to 
be taken as non-existent (e.g. the diagonal set of the supposed bijection f).

NFU 'solves' this problem by having more atoms/pairs than sets, but this looking at the cardinalities involved - is obscure, because this means just too many atoms or even not every atom having a singleton! The third category of objections asks us to reconsider some ontological alternative:

(vi) Suppose there is no set of all sets but a realm set theory is talking of. There is no need to take it as a set; supposedly everything useful can be said talking of sets only. There may be subparts of this realm (e.g. the part where the ordinals are), but again this realm/part-relation may be something like mereological composition. There is no need to have a theory of proper classes (as a theory similar to set theory).

(vii) Once we allow for more than finite sets not every condition defines a set - remember that Naïve Comprehension is consistent on the finite sets! The limits of restricted Comprehension (like in ZFC) are just a 'failure' to commit us to even more infinite sets. That doesn't seem so bad. Given the set of finite sets, Naïve Comprehension and (restricted) Comprehension come to the same thing. As Naïve Comprehension means trouble only with infinite sets this again may be rather a problem of the infinite. In the infinite ZFCComprehension (i.e. Separation) doesn't seem too bad.

The main alternative to a universal set seems to be the idea of an unbounded sequence of ever higher cardinalities. The idea of classes provides just an intermediate halting place before collecting them to proceed further!

Can we really understand an incomplete universe? The short reply that in reading a book on it, writing a paper on it and having a discussion we obviously understand the position we are attacking is too simple. Obviously one can retell what some authors have published or said about 'absolute infinities' or an 'incomplete universe'. In a sense of "understand" we understand the strangest stories that lack coherence, at least up to a point. Around that elusive 'point' of not coming to terms with a story or theory we are at a loss what somebody is writing or talking about. We are at a loss - we may conjecture - because what we are told goes massively either against our standards of coherence or against our understanding of involved core concepts. This conjecture is still quite vague. And, with respect to 
paraconsistent set theories, we cannot appeal here to strict standards of consistency against dialetheists without begging too many questions.

Metaphysics in analytic philosophy traditionally (starting with Frege) could be seen as deriving from semantics or an account of the truth of statements/propositions. Frege assumes concepts as entities in their own right and with their peculiar feature of being 'ungesättigt' (having a gap to be filled by some argument), because he needs this ontological assumption in his account of assertions/statements and their cohesiveness. With the increasing interest in ontological questions in their own right metaphysics nowadays if often seen as independent from semantics, not to speak of epistemology. Metaphysics in this newer tradition advances as axiomatic ontology. "Axiomatic" is meant in theories of this type in a sense close to the sense of laying down axioms in logic or mathematics. Axioms may be useful (say in applying mathematics in science), but foremost are stipulations concerning either the concepts or the entities contained in them. As Carnap saw 'no morals' in logic, so that according to his principle of tolerance every system had a right to be developed, so the axiomatic ontologist may see no morals in ontology so that there are no restrictions on ontological postulates. In the light of this approach one might consider any set theory as just another axiomatically founded ontology. The axioms just being its introduction.

Carnap may well have been wrong about conventionalism in logic, at least in the sense that human reasoners follow one specific logic (or a narrow range of logics) and in that the human language faculty may contain one specific logic (or a narrow range of logics). In a similar way stories and accounts about the possible furniture of the universe may be interesting in their own right, but given even a mild realism, there cannot be just any entity proposed by some story or ontological account. And given, further on, some mild evolutionary account of our cognitive faculties our human conceptual system cannot be neutral with respect to ontological theories.

The debate over incomplete universes vs. inconsistent totalities might thus be put: Does our conceptual system allow for them?

The difficulties which one may have with the mere postulation of some axioms may point to some more general methodological lessons in metaphysics. Something seems to go wrong here with axiomatic ontology. From the point of view of the Fregian tradition, which put semantics before metaphysics, axiomatic ontology has gone too far. Notwithstanding its limits or shortcomings the linguistic or conceptual turn of (analytic) philosophy is still endorsed by a proponent of this tradition. Postulating relations works in science in as much as the postulates are borne out by the thus established (scientific, experimental) practice and theoretical 
framework. Definitions are not refuted by evidence, but some definitions turn out to be useless or to be incoherent with other parts of a theory. As has been noted often: Conventions (alone) do not deliver truth(s). We understand what it means to tell a story. We may conceive of such a fiction even in terms of images what the world had to be like if the fiction was true. We employ our imagination thus when reading a book others do that when producing a (fantasy) movie. No one, however, can imagine the Russell set or the round square in that way.

To decide between ontological proposals we need criteria to judge their respective merits. These criteria may take up the tenet for which the respective ontology was developed. Alex Oliver (1996, pp.2-13), discussing the metaphysics of properties, proposes to look at an ontology's achievement in conceptual analysis, in as much as ontologies are introduced to account for the function and content of expressions. To understand what a name is one introduces referents, for example. Following such an analytic procedure (types of) entities are introduced which, according to the analysis, account for our use of language. And no other entities are to be introduced, as ontologies have to be economical.

With respect to ontological economy one may distinguish between ideological economy and ontological economy proper. Ontological economy proper concerns the number of introduced types of entities. Ideological economy concerns the number of undefined basic concepts of a theory. The less undefined concepts a theory has, the more concepts have to be defined, and the more inferential links will be present in the conceptual system of the theory, which therefore shows a higher degree of systematicity (cf. Goodman 1943, 1949, 1972, pp.275-355).

Now, we can increase the ontological economy proper of a theory if we introduce undefined operators and relations instead of basic types of entities (say, if we do not define "necessary" and so forsake the introduction of possible worlds). And vice versa: We can define former undefined concepts by introducing new types of entities to occur in their definitions. This is even true of methodological concepts (say, if we substitute "being a relation" by ordered pairs or tuples). How can we decide then the degree of simplicity of an ontology?

One constraint is the provision of fruitful conceptual analyses of concepts we employ in our ordinary thought and discourse. ... Here, as in all philosophical inquiry, we must adopt the method of reflective equilibrium, balancing the demands of theory against the preservation of commonsensical beliefs. .. [O]ne cannot hope to defend a metaphysical theory by constructing knock-down arguments against each of its competitors. There are numerous ways to trade off ideological and ontological economy and to balance these theoretical benefits against the preservation of common-sense belief. One can only hope to draw up a cost 
and benefit score sheet, it being a very real possibility that there will be ties for first place. (Oliver 1996, pp.4-5)

Systematicity (and thus a higher degree of ideological simplicity) yields more explanatory power, since less fundamental principles (those containing the undefined basic concepts) have to be used. Conceptual analysis provides a decrease in the number of undefined concepts. ${ }^{52} \mathrm{We}$ explain a fact by conceptual analysis by being explicit about the concepts which are used to describe the fact (cf. Oliver 1996, p.6). So a high number of definitional links increases explanatory power. Therefore, one usually may prefer higher ideological economy over higher ontological economy proper, as regularly theories are chosen for their explanatory power (cf. Thagard 1978, 2000). Thus one will accept those (types of) entities which are introduced in building up the framework of such a theory.

One might consider ideological economy as a meta-constraint on ontologies as they are to be embedded into wider theories, which have to compete in explanatory power. Another meta-constraint could tie an ontology to a broader scientific perspective like nominalist scientific realism etc. We will focus here on two basic criteria for ontologies themselves. One may keep ideological economy as an important desideratum in mind. Generally, however, we may prefer the foundations of mathematics to be as neutral as possible with respect to controversies between concrete scientific theories.

These considerations lead to one criterion of a successful ontological theory:

(O1) Legitimation by Conceptual Analysis

Exactly those (types of) entities are to be assumed which have to be introduced by the best conceptual analysis of the target domain of concepts.

(O1) has to be supplemented, however, by a second criterion:

(O2) Epistemological Constraint

An ontology which introduces (types of) entities has to contain a theory how we know of these entities.

52 One need not subscribe to a highly controversial theory of concept definitions to have some form of conceptual analysis. The argument presupposes only that some form of conceptual analysis is possible (cf. Jackson 1998), where conceptual analysis is even viable in theories which deny that there are enough definitions providing an analysis into necessary and sufficient features, as long as we allow for any inferential links (cf. Bremer 2008). One may even speak of 'analysis' simpliciter as one stresses that this analysis yields substantial insights, often denied to 'mere' linguistic or conceptual analysis (cf. Williamson 2007). 
This constraint will not be endorsed by someone following a 'pure' axiomatic approach to ontology. Giving up $(\mathrm{O} 2)$ on the other hand means giving up the approach to ontology which ties it to our conceptual system and our use of language (as any theory of these will have to contain a theory of how we refer or 'hook up to' entities of some kind). Not subjecting a conceptual analysis to the epistemological constraint seems to miss that the target of analysis are our concepts and linguistic abilities. Any analysis of our concepts should contain a part explaining how we can use these concepts or manifest our knowledge of their proper employment. Even if one does not follow the strict agenda of Michael Dummett (1991) and his adherents any theory of our concepts should contain a part explaining our use of these concepts in our engagement with reality and other speakers. If our possessing some concept is reduced to some type of entity (as outlined above) then a comprehensive theory of our concept possession should contain how we can stand into contact with entities of this type or can know of them.

Criterion (O2) seems to stack the cards in favour of some causal theory of knowledge or access. This need not be so. (O2) can be weakened to the requirement of giving an account of how we can make justified statements about the entities in question. Taken thus, Plentitudenous Platonism, which claims that we by developing consistent theories have justified beliefs about abstract entities, passes this test, as noticing consistency is taken as faculty independent of an elucidation of consistency in model theory or logic (cf. Ballaguer 1998, pp.48-75).

David Lewis' modal realism for all its ideological economy, relying just on classes and all individuals (everywhere), is mostly rejected by its blatant failure to give a convincing answer to $(\mathrm{O} 2)$, as Lewis postulates the absence of any access connection between us and (other) possible worlds (denying both spatial and causal access). This - as with Plentitudenous Platonism - may be too quick. Lewis may well argue that justified belief in modal realism is enough if there are just these worlds.

There may be more conditions one may like to lay down for ontological theories. On the other hand the burden of outlining other conditions for understandable and acceptable ontological theories lies with the axiomatic ontologists.

Let us consider four set theoretical options in the vicinity of universality introduced earlier [in chapters I \& V] in face of these conditions. We have to turn to some analysis of the involved concepts. 


\section{(1) Noneism}

The following discussion will be concerned only with noneism (i.e. with a theory subscribing to the noneist principles (P1) - (P6) in some form) and Meinong's thesis of non-being ('Außersein'). ${ }^{53}$

David Lewis (1990) complained against Routley's use of two ways of quantifying, one time with existential impact, one time without. The mere occurrence of two types of quantifiers may not be the core of the problem. Non-committal quantifiers have become a common tool in modal and Free Logics, usually in combination with an existence predicate. The concern behind Lewis remark on the quantifiers points, however, in the right direction: If "there are" is not committal in any sense (i.e. not even to possibilia, as it is committal in many Free Logics), what does it mean after all? We (i.e. we who are not noneists, yet) run against a way of talking defying our understanding. Our first problem is not that we do not agree with the noneist, but that we simply do not understand what to disagree about.

The distinction between existents and possibilia is a clear one in modal semantics. Noneism claims even less being than possible being, but still uses the forms of "to be". What an object beyond all being is supposed to $b e$, is beyond us non-noneists, and our human concept of object, one may suppose. Ryle challenged Meinongianism of abusing the expression "object":

...the important sounding word 'object' never did have any other positive function than to be a synonym for 'subject-matter' or 'remark-topic' (1973, p.257).

That thoughts have representational content no one will deny, that thoughts have 'objects' beyond those representations is a far more substantial thesis and no obvious consequence of the observation on representational content. For noneism, however, everything depends on Holmes not just being a file of propositions attached to the expression "Holmes", but being a unified something (an 'item'). Noneism seems to trade on the almost imperceptible shift from 'content of a thought' (easily identified by citing the representation employed in the thought) to 'object' (as something beyond

53 Thus even if there are major problems for noneism that need not tell against Dale Jacquette's or Terence Parson's theories of non-existents, which they claim go back to Meinong. The same applies to work done in the vicinity of Gegenstandstheorie (e.g. the papers in Haller 1995). In my eyes though, Routley is right in seeing his version of noneism as a proper articulation of Meinong's main theses. Even if that is not so (i.e. if Meinong is misrepresented by the noneist), the challenge posed by noneism is worth considering in its own right. The reference to Meinong then has only a motivating function.. 
its representation). As Priest stresses: '[A] noneist accepts objects of thought as genuine, not just as linguistic simulacra' (2005, p.42).

The noneist's "there are" posits a "there" which we cannot locate and are not allowed to locate on pains of importing being into the theory. And "cannot locate" means here not just without location in space and time, but without being placed in any 'realm' like Frege's or Popper's 'third world' of thoughts or abstract entities. Chisholm (1973) once appropriately called the noneist's objects 'homeless' as they neither are in the concrete realm (the universe) nor beneath the Platonic forms. The noneist's "there" is equivocal when applied to ordinary objects, which exist somewhere, and non existing objects.

The Independence Thesis itself seems, at least in the noneist's reading, quite questionable. BEING-RouND in case of the non-being round square cannot be the same manner of BEING- ROUND like in the case of a penny coin. BEING-GOLDEN in case of the non-being golden mountain cannot be the same manner of BEING-GOLDEN (i.e. having some physical structure) like in the case of the gold bar. All general terms seem to become ambiguous here!

One may represent states of affairs involving existing and non-existing objects, and properties in the same format, say:

(1) <The golden mountain, Golden, $1>$

(2) $<$ Peter's gold bar, Golden, 1>

This, however, is only a formalisation at the level of an ontological theory. What we also need is an account what having a property comes down to. In case of physical objects we have such accounts (like trope theories or property realism). These theories cannot apply to non-existing objects. How, then, are they to have their properties?

Again, describing an non-existent object as having some property does neither explain how it has that property, nor does it explain how this succeeds in the object 'being there'. Usually speaking about something does not make it the case. Does this distinction have any application in case of non-existent objects? Even describing a non-existent object other than it was introduced in its characterization need not be a matter of falsity, since non-existent objects may be inconsistent. Even defining a non-existing object as "consistent" leaves it difficult to distinguish false claims about an object from changing the subject or extending the objects definition/characterization.

Thus saying

(3) '[V]ery many objects do not exist in any way at all'

(Routley/Routley 1973, p.227). 
makes no more truth - or maybe sense - than saying

(4) The Arch-Supervisors co-inhabit collectively the hidden dimensions of Gaia.

Noneists can hardly claim their descriptions and postulates to be acceptable to their audience by being evident. This applies as well to Routley's distinction between reference (the supposedly bad idea behind most of today's philosophy of language) and aboutness (the supposedly benign and non-committal relation between names and non-existents, inter alia). Instead of the Referential Assumption (RA) Routley endorses something like an Aboutness Assumption:

(AA) Every singular term is about some kind of being.

On first sight (i.e. before becoming a noneist) this is a distinction without a difference. Especially if one holds that reference - as shown by definite descriptions - does not require a causal connection, there is nothing that sets aboutness apart from reference.

To defend noneism it is not enough to stress that we understand talk about fictional objects and thus talk about non-existents. ${ }^{54}$ This understanding only counts for noneism if noneism was the only or best account of fictional objects, which, of course, is contested. We understand what it means to tell a story.

Meinong (cf. 1904, $\S 2-3$ ) argues in favour of noneism by pointing to (i) thoughts always having 'objects' as content, (ii) the countability even of things we know not to exist, and (iii) negative existence statements. (iii) has been dealt with by Russell's or similar theories of descriptions and elimination of non-referring terms in favour of variables and predicates. (ii) ultimately reduces to (i) as the counting concerns objects of thought. So Meinong's main argument claims that the intentional structure of thoughts (and indirectly sentences) has to be accounted for by positing objects of thoughts, every thought dealing with its objects. Meinong thus shares with the so-called 'semantic tradition' (cf. Coffa 1991) the thesis that thoughts have objective contents, which can be shared. But whereas the semantic

54 Both Frederick Kroon (2008) and Daniel Noolan (2008) in their criticism of Priest are not clear on this point. Kroon bases his criticism on a problem with the properties of the fictional character Gandalf, and sees this as 'an example of our apparent ability to talk about what does not exist' (p.199). Noolan says, 'Non-existents are also very useful as possibilia' (p. 191), which for the noneist they are - often enough - not: They have not possible being, but no being at all. Meinong and Routley are perfectly clear on that. Priest employs possible (and impossible) worlds in his version of noneism, which complicates matters a lot, and which invites understanding non-existents as possibilia, as they exist in some 'possible world'. On the other hand he is outspoken against a possibilia account of non-existents, for example: 'I deny that quantification over something requires it to have any form of being' (2008, p.214, Priest's emphasis; similar: Priest 2005, p.14). 
tradition sees these contents as sentences or their meanings (i.e. as representations or abstract objects) Meinong uses a generic concept of 'object' and binds it to the Independence Thesis. Even if the positing of objects of thoughts was required by a conceptual analysis, say of propositional attitudes, the further step is an addition [see $\$ 4$ above].

There are other theories of intentionality and intentional objects besides noneism. Making a de re/de dicto distinction or employing a Free Logic accounts for the failure of quantifying into intensional contexts and related phenomena. Representational theories, which take propositional attitudes either as relation to representation of the language of thought or representation of a public language, provide a model of the attitudes without too much ontological commitment. The goal of ontological scarcity or simplicity - if that is a goal to adhere too - thus does not favour noneism over these theories, at least not to a degree which makes dealing with its extravagancies worth while. The crucial - and in my eyes not met challenge to noneism demands arguments from the noneist which show that in the critical cases of non-existents "thinking of $a$ as $F$ " is not reducible to "having a representation: $\mathrm{F}(\mathrm{a})$ " (or a representation belonging into an equivalence class of translations or synonyms of: $F(a)) .^{55}$

Noneism engenders epistemological problems both in accounting for reference or the substitute for reference, and for the truth of non-existential committal theories: Are there non-existent truthmakers for facts about nonexistents? Frege and other Platonists speak of a relation of grasping a thought. Relating to pre-existing thoughts or other abstract objects by such a faculty of grasping is one of the major and controversial topics in the philosophy of mathematics. In this case, however, the problem is simpler than the related problem for noneism. The Platonist has/postulates a 'realm' (a Fregean 'Drittes Reich'), which is targeted by the grasping. Non-existent entities are not anywhere. Their being 'entertained' thus - if not just reducible to the representation of sentences - is far more elusive than Fregean grasping. Meinong himself (cf. 1904, pp.10-12) speaks of 'grasping' a 'pre-given' ('vorgegeben') object, then rejects talking of 'quasi-existence' and settles to the thesis that the contrast between being and non-being arises only with (complete) states of affairs, not single objects, which are, therefore beyond being. This, however, threatens to reduce objects to thoughts about objects in assumptions (presumably sentence like representations); and Meinong's states of affairs ('Objektive')

55 Priest (2005, pp.58-59) criticises representational accounts of propositional attitudes, without putting weight on these reflections. His main criticisms concern the problem of quantifying in and the problem of introducing an equivalence relation on representations without invoking the objects they are about. The first may be answered by some Free Logic account (with possibilia, say), the other by some form of inferential role equivalence between representations. 
are (sometimes) said to be non-existent themselves (cf. 1904, p.6)! The non-existence of states of affairs is also very explicit in Routley and Priest. So with respect to grasping a non-existent nothing is gained here. Meinong ultimately declares that every object could be known, and everything that could (!) be known has 'givenness' ('Gegebenheit', p.20). 'Given where by whom or what?', one inclines to ask. Later Meinong (1921, p.20) stresses that grasping is something 'ultimate, indefinable' ('ein Letztes, Undefinierbares'). The faculty of grasping an non-existent object, so, is a theoretical postulate in Meinong's theory.

Priest also at one point (2005, p.142) posits a faculty of 'pure intention', which is able to bring us into contact with any object whatsoever. He provides no account how this faculty works in detail. Further on, he claims phenomenological evidence for acts of object access:

[W] hen one fears something, one has a direct phenomenological experience of a relation to the object of the fear. And the phenomenology is quite independent of whether or not the object actually exists. (Priest 2005, pp.57-58)

As often with phenomenological evidence claims this one seems open to disagreement: Does one not rather experience the content of one's fear, whether or not it is (semantically) linked to an object or not? What would it be like to experience an object simpliciter, in any case? A representational theory seems closer to the phenomenology.

Priest ultimately tries to soften the access problem by moving his version of noneism closer to fictionalism and RTF:

The properties of Sherlock Holmes may be just as a priori as those of 0 . In both cases, we characterise an object purely by fiat. We know a priori that the object so characterised has those properties (at certain worlds), and this is so whether the characterisation is provided by what is told in Doyle's novels or by the Peano axioms. (Priest 2003, p.9; cf. 2005, pp.145-48)

In fact, somewhat against the spirit of Routley Priest's noneism is more a fictionalism than noneism. Priest - like the fictionalist - regards fiction as false. Priest also regards it as possibly true (i.e. true in the worlds of fiction). If $\alpha$ is any claim about the non-existence entailing properties of a mathematical entity, the noneist should claim $\alpha$ to be simply true, whether the mathematical entities are existent or not (as they are not, of course, for the noneist). Priest has to say that $\alpha$ is true at the worlds in which the entities exist, i.e. has to take $\alpha$ as possibly true. At the actual world it is true: $\diamond \alpha$. Mathematical statements become intensional. Priest so endorses a revisionist theory not only of mathematical ontology, but of mathematical truth as well. If that saves noneism then it does so only at the price of moving towards fictionalism and trading in the problems of fictionalism. As Priest uses the strategy of entities at some world not just for 
mathematics his theory - despite (Priest 2008, p.214; 2005, pp.14, 42) looks rather like a theory of possibilia in a paraconsistently extended Free Logic than like Routley's or Meinong's theory of non-existent items. ${ }^{56}$

The criterion $(\mathrm{O} 2)$ of coming into contact with entities of a proposed type seems questionable if an ontology itself entails that there is no (causal) contact to these entities. Noneism here resembles those versions of Platonism which deny that abstract entities interact with the physical world. As noneist 'items' are not existent requiring access seems requiring too much. But this is the basic problem with noneism again: 'items' are said to be 'there', thus are not nothing, or - again - the whole theory seems incomprehensible. A fictionalist invents stories and thereby accesses fictions easily. Noneism claims to be something different from fictionalism, but it seems it cannot.

Noneism is incompatible, further on, with any form of semantic externalism, as externalism typically invokes some causal mechanisms of hooking up concepts and lexical items to properties in the world. Noneism's attack on the Referential Assumption has to apply to properties and 'content' as well. As many epistemologists and semanticists support at least some version of externalism noneism calls for a far and wide revision of these fields as well, respectively is put into doubt by the success of externalist accounts. Externalism has its own problems with fictions, but any progress here helps fictionalism - again - and not noneism.

Noneism in denying being to inconsistent objects is too weak as foundation of paraconsistent set theory, since sets - unless you believe in noneism are usually taken to exist (even if not in space in time). Noneism certainly is no version of realism.

Noneism is either a version of a substitutional understanding of individual expressions such that we can use a non-referring expression and tell a story in which this expression occurs (for example a story about a unicorn) in which case intensional contexts are to be understood in some way paratactically in the tradition of Carnap's intensional isomorphy or Davidson's saying that - or noneism is not comprehensible at all.

56 There are a few additional problems with circularity for Priest's account, it seems. Firstly, possible worlds are considered to be non-existents as well. So what can it mean that all non-existents exist at some world? Priest's version of Characterisation has worlds 'realize' other non-existents and their properties. As worlds are taken as nonexistents, where do they get realized? Or can they realize without being realized themselves? Worlds seem to exist at themselves. Secondly, discussing the question how non-existent mathematical objects can be used to describe reality (the actual world) Priest claims that the physical quantities and the mathematical quantities 'have the same structure' (2003, p.12; cf. 2005, p.150-51). But a structure is a paradigm case of something abstract, thus non-existent for Priest. A regress seems to ensue. 


\section{(2) Incompletability}

One of Cantor's arguments in favour of transfinite numbers was the naturalness of extending the successor function and the taking of unions beyond the finite. Once one has understood that the finite sets as a collection have to be taken in a set as their collection, one naturally extends the order $\omega$ of this set to $\omega+1, \omega+2 \ldots$ Who admits the ascent to ever larger natural numbers cannot resist the ascent to $\omega$ and above. (Therefore strict finitism attacks already at the first ascent.)

The picture behind the iterative hierarchy seems to contain the idea of indefinite extensibility. Whatever axioms of large sets we add we can imagine the 'process' of set generation going on from there beyond these intermediate halting points. Incompletability seems to be built into this picture of sets. No collection provides a natural stopping point to any further 'construction'.

Can we comprehend incompletability? Properly speaking - as we are not talking about processes of reasoning or construction, but about ontic structures: Can we comprehend an incomplete universe?

The concept seems to be beyond comprehension as it is logically true: Everything that exists exists. Whatever is in the hierarchy is. Thus - we seem to be forced to go on - it is somewhere. Thus this somewhere cannot be incomplete.

The concept which causes these conundrums is the concept of quantification. Once we subscribe to the idea that quantification requires a domain we are set for trouble. But how could QUANTIFICATION and DOMAIN OF REFERENCE ever become decoupled? Does our conceptual system allow for - or even contain - a concept of quantification without domain?

Raised in standard meta-logic we are accustomed to assume not only a domain, but we see this domain as another thing, which then 'of course' has to be contained in its (i.e. another) domain.

The INDEFINITE is a crucial concept in Hilbert's finitism. Speaking of an indefinite object seems to allow having an object of arbitrary size without committing oneself to quantifying over a domain of infinitudes. Hilbert also invented and used his $\varepsilon$-operator to this purpose.

Hilbert's finitism (Hilbert 1925) was not directed against the idea of the ever larger infinities of 'Cantor's paradise', but was inspired by the idea of secure foundations for talk of infinity, where in these foundations (considered as meta-logic) the notion of the infinite was not to be presupposed. What is interesting even for the critic of infinities in Hilbert's finitism is his method of trying to work around the commitment to actual infinities (cf. George/Velleman 2002: 147-72; Shapiro 2000: 158-65). 
Hilbert wants to justify set theory and Cantor's theory of transfinite numbers by using only finitary arithmetic in the meta-theory. Finitary arithmetic includes equations and their truth functional combinations. Also sentences with bounded quantifiers, like " $\forall \forall x<120)$ ", are admissible. Any combination of such sentences is effectively decidable (by dealing with finitely many specific numbers and their properties). Now, to include some generality (i.e. to be able to make general statements like the commutativity of addition) Hilbert introduces schematic letters: $a, b \ldots$. One can thus express

$$
\text { (CA) } a+b=b+a
$$

Hilbert considers a statement like (CA) to be finitary! The idea is: Which ever specific numerals we choose to replace " $a$ " and " $b$ " the corresponding statement will be an acceptable (decidable) finitary statement.

Whereas in standard logic one typically reasons

(UG) For some arbitrary $\mathrm{x}$ : F(x)

$$
\begin{aligned}
& \mathrm{G}(\mathrm{x}) \\
& \text { Thus: }(\forall \mathrm{x})(\mathrm{F}(\mathrm{x}) \supset \mathrm{G}(\mathrm{x})) \quad \text { since } x \text { was arbitrarily chosen }
\end{aligned}
$$

the finitary reasoning is different. (UG)-like reasoning infers to the totality of the domain. Finitary reasoning rather argues:

(FG) The following proof scheme is valid for any instance:

$$
\begin{aligned}
& P_{1}(a ́) \\
& \ldots \\
& P_{2}(a ́)
\end{aligned}
$$

Thus: $\mathrm{P}_{1}(\mathrm{a}) \supset \mathrm{P}_{2}(\mathrm{a})$

Nothing is supposed about a totality of objects. It is rather provided a scheme to turn the assumption $\mathrm{P}_{1}(\mathrm{a})$ for any given or thought of individual term into a proof of $\mathrm{P}_{2}(\mathrm{a})$. One might express this as the dialogical challenge 'Once you name the object, I will provide the proof that it is well-behaved as well.'

Employing the Wittgensteinian distinction between saying and showing one can understand the distinction between (UG) and (FG) as having (UG) saying what (FG) only shows, where, of course, Wittgenstein, who after 1929 took a position close to the use of schemata by Hilbert or Skolem (cf. Marion 1998), would add that what (FG) shows cannot be said at all, since there are no completed infinities. 
Since no totality of objects to be quantified over is presupposed one need not assume even that there is a totality as the infinite set of natural numbers. One just claims that theorems can be proven for any number expression that someone comes up with. Since this is a general claim about forms/schemata of theorems - what else should one wish for in arithmetic?

Apart from the difficulties with Hilbert's larger program (like the incompleteness theorems, especially Gödel's Second Incompleteness Theorem) finitism in this sense has its own difficulties. What to think, for example, about the negation of schemata like (CA)? One might think they are equivalent to sentences or schemata with an unbounded existential quantifier. Hilbert thus sees them as 'transfinite propositions', i.e. as illegitimate in finitism. ${ }^{57}$

Nevertheless one may take up the idea of schematic representation. Do we have some additional faculty of schematic abstraction? That needed some

57 And even excluding negations of schemata like (CA) seems to leave one with primitive recursive arithmetic (cf. also Tait 1981). Remember that the primitive recursive function do not include $\mu$-minimization. Primitive recursion comes down to bounded quantification. To have the usual means of logic available Hilbert allows these non-finite formula in, but considers them as 'ideal' (i.e. devoid of respectable finitist content). Formulas are only considered in their inferential role. The formulas themselves can then be taken as the new (material) objects of this reasoning. Thus finitism gives way to formalism.

Three side remarks may be in place here. The first concerns strict finitism as a radicalisation of finitist ideas. In case the universe is complete there are two options: it is finite or it is infinite. Most set theories we have considered - in any case all used in mathematics - are committed to many different infinities. A universal set within any of them or an updated version building in $U$ has $U$ as infinite set. For the strict finitist, on the other hand, $U$ as well as all other sets have to be finite sets. This leads to some more peculiar features of strict finitism (besides, say, its limitations of self-reference by having limited resources of coding).

The second side remark concerns the idea of indefinite extensibility in relation to Gödel's Incompleteness Theorems. By Gödel's Incompleteness Theorems we know that unfortunately standard consistent set theories are negation incomplete in arithmetic. When we start with axioms of larger cardinalities we establish stronger set theories. As stronger theories can prove the consistency of weaker theories we have in a theory $\mathbf{Z F C}^{+}$some theorem stating the consistency of $\mathbf{Z F C}$. This theorem - by way of Gödelization - has to be an arithmetical truth not derivable before. Thus even standard arithmetic seems extensible by this procedure. The extensibility of arithmetic, however, cannot be indefinite. There are not enough arithmetical truths. If an arithmetical property is modelled by a number set there can only be $\aleph_{1}$ many questions: for $\aleph_{0}$ many numbers we can ask whether they are in one of the $\aleph_{1}$ many sets. Arithmetical extensibility is limited. If we add indefinitely many axioms of large cardinalities the preconditions of Gödelization finally give in as well.

Thirdly, one should not confuse the incompletability envisaged for the 'universe' of sets with 'productivity' in computability theory: productive sets can be extended, but as this extending concerns recursive enumerability the extended sets have a definite cardinality in the lower infinite. 
explanation. The ineffability problem raises its head. Postulating a basic faculty of schematic intuition deserves - at least - not more credit than postulating a faculty of intuiting $\mathrm{V}$. One may ask in general what understanding a schema - especially one over an arbitrarily extensible 'ream' of individuals, not over countable many well-formed formulas of a specified language - comes to. Is it not just to understand that some schematic representation is true/well-formed/valid for all its specifications? In understanding the schema we seem to have access to the domain of its instances (respectively the domain that these instances are talking about). In this case we seem to have a strong intuition in favour of some Domain Principle.

Dropping the universal quantifiers and reversing to substitutional schema or rules instead of axioms in set theory does not help either, as there are neither enough names around for substitution (names being countable anyway) nor do we get rid of embedded universal claims so easily: just look at the Axiom (schema) of Replacement.

So, the idea of an incomplete universe does not far well in face of condition (O1): our concept of quantification seems to be tied to a Domain Principle, which cannot be circumvented by talk of the indefinite or schemata. The incomplete universe resists stable quantification. If the universe is extensible and grows - between different times? - universally quantified set theoretic sentences shift in meaning - at least in their truth-conditions as their domain of reference shifts!

Another way to get rid of or ignore the Domain Principle may be to rest one's understanding of quantification solely on the semantic rules for quantifiers (i.e. introduction and elimination rules), similar to so-called 'anti-realistic' theories of meaning (cf. McGee 2006). Semantic rules may be (partially) constitutive of meaning, at least for logical vocabulary, explicitly definable. Understanding the rules for " $\forall$ " and " $\exists$ ", however, involves somehow - it seems to me - thinking of the source where the instantiation terms or assigned values of variables come from, i.e. a corresponding domain.

And even if we drop the Domain Principle we have not made the idea of a thought independent but at the same time incomplete universe of sets any clearer. In the context of set theory no further objects can come into existence. The status of the universe itself as an object of incompletable nature awaits elucidation. The whole idea has an radically anti-realistic flavour, foreign to the set theories discussed here. 


\section{(3) V as entity sui generis}

Given the difficulties in understanding an incomplete universe and the fundamental role of a Domain Principle, why don't we just talk about V without assuming it to be the value of a bound variable? This appears reasonable as doing otherwise land us in an incomprehensible framework of indefinite existents.

Assume we do not give up on the infinite, whether we are Platonists or fictionalists or whatever else. There are then infinite collections. Comprising within them all of a kind not collectable itemwise by finite beings. We collect them using our concept COLLECTING. If we talk about the $F$ s we naturally assume that there is a collection $F$ where they are in. Cantor's Domain Principle expresses this idea that the $F$ s we quantify over or talk about can be collected into a totality. Sometimes the totality has to be of another type to avoid antinomies (e.g. in the set/class-distinction). As there seems to be no limit to this procedure we always progress to a wider domain. ${ }^{58}$ The Domain Principle thus enforces the idea of the incomplete universe. A domain is added to the objects, giving a larger domain, which is added to the objects - and so on.

Unless, that is, we meet a fixed point in this progression. Informally, the totality of things to be thought of or to be talked of can be thought or talked of: it belongs to the very domain it defines. Thinking of 'the domain $\mathrm{x}$ is in' applied to it leaves us at it. Thus it may be called a fixed point of the Domain Principle.

Is $\mathrm{U}$ of this type? Having $\mathrm{U} \in \mathrm{U}$ requires several other adjustments in set theory. And they do not come cheaply - up to inconsistency.

As intuitive as the Domain Principle may occur to us, leading us up the ladder of the indefinite may be too much, as we have seen above. We might accept that the whole construction has a limit: a collection beyond further collecting. There lays the naturalness of Limitations of Size: There is one size too big to be collected into a set. This collection better not be the set $\mathrm{U}$ to avoid severe complications in set theory, otherwise rather intuitive. So one may see the idea behind Limitations of Size without endorsing NBG or MK, or any other set/class-theory. Nothing is gained by having (several) classes. With a collection of classes the question of their collectability immediately arises.

\section{The single limit object $V$ might be different.}

58 Recently Rayo and Williamson (2003) and others have argued for 'unrestricted First-Order languages', i.e. for quantification without a domain. The formal proposal, however, must employ SOL and a richer meta-language for which similar problems arise. One may also consider the employed SOL as critical and problematic (cf. Weir 2006). Unrestricted quantification is only unrestricted beyond an object/meta-language distinction. 
If that limit object $\mathrm{V}$ exists - neither a set, nor an extended set like an inaccessible cardinal, nor a class - ZFC is consistent. And if our intuitive notion of set rather endorses the General Continuum Hypothesis we add it as well: ZFGCH is consistent, if V exists. Our notion of set suits V, and vice versa.

This conception of $\mathrm{V}$ as collecting all the sets but being a special limit object may correspond better to our concept SET than taking set theory just as the realm up to the first strongly inaccessible cardinal. [The Cantor quote setting the theme of this book may illustrate this perspective.] Someone might argue that our concept SET takes us thus far, but that there are other mathematical objects and theories (especially those of large cardinals, measures etc.), which pick up the baton where ZFC hands it over. Although this sounds like a nice division of labour, the large cardinals are too set-like to provide a natural boundary to our concept SET, supposing it to fit to $\mathbf{Z F C}$ in the first place. $\mathrm{V}$ is a stop point, the first inaccessible cardinal is not. And large cardinals - again - give rise to the question where their hierarchy is collected in, inviting and requiring $\mathrm{V}$ or some V', landing us again in an incomplete universe. $\mathrm{V}$ is not an incomplete universe at all: although we cannot walk, count or 'powerset' us up to it, $\mathrm{V}$ contains all sets; they are not in the making, there are no processes of indefinite extension going on. In this respect $\mathrm{V}$ as an object at the limits of thoughts differs from the row of experiences discussed by Kant in the Critique of Pure Reason: Kant traces the antinomies to their common error of taking the series of experiences, which is only given piecemeal and prospectively ('aufgegeben') as a 'given' totality. As experiences are obviously under temporal construction their series can never be united - by whom? In an experience? Sets, in contrast, are not (temporally) constructed and thus should be collectible in a unity. Thus far we are carried by the Domain Principle. At that limit we 'simply' have V as an object, and stop adding it to a domain.

Our concept of SET may force stronger set theoretic axioms on us. This shows, however, not the incompleteness or growing extension of $\mathrm{V}$, but the incompleteness of a theory like ZFC. Urelements and $\varnothing$ have no members, but are members; sets have and are members; V has members, but is not a member: it occupies a slot in conceptual space. ${ }^{59}$

In the light of the two criteria (O1) and (O2) we may say: our idea of V is an idea contained and connected to our concept SET. The special nature of $\mathrm{V}$ is forced upon us by the unfeasibility of the idea of an incomplete or thought independent but growing set theoretic universe. We know of $\mathrm{V}$ by

59 The slot of neither having members nor being a member finds no existing filler, if there is not David Lewis' atomless, uncollectible 'gunk'. 
the picture we have of the iterative hierarchy and the structural relations between the ranks.

This conception of $\mathrm{V}$ follows some intermediate path between the two ontological traditions in analytic philosophy. On the one hand there are reasons of conceptual analysis why V suits our concept SET. On the other hand some peculiar postulates need to be laid down for V. "V" is a rigid designator naming an entity which does not belong to some domain of quantification, although all other entities and referents of names do!

The major difficulty here would be to allow for a level or form of metatheory when talking about $\mathrm{V}$ which is outside of any formal system. That way may lay ineffability or some version of ontological semantic mystery!

Comparing noneism, the incomplete universe and the thesis of $\mathrm{V}$ being an entity sui generis, the third idea comes out best in its combination of conceptual analysis and axiomatic ontology.

If we do not want to follow this path and acknowledge the problem of talking about $\mathrm{V}$ outside of $\mathbf{Z F C}$ a paraconsistent universal formal system seems to be obligatory. A system that integrates theory and meta-theory.

\section{(4) Inconsistent Platonism}

As mentioned before Ballaguer (1998) defends Plentitudenous Platonism as an option in the philosophy of mathematics, since Plentitudenous Platonism can meet both criteria $(\mathrm{O} 1)$ and $(\mathrm{O} 2)$. Platonists like Frege in his Grundlagen der Arithmetik (1884) argue at length that Platonism is more natural and more embedded into our conceptual intuitions than any rivals like naturalism, psychologism or constructivism. Although we cannot get into direct (causal) contact with abstract entities if we possess no faculty of logical intuition, we can have beliefs about them which may turn out to be true. If all consistent abstract realms exist and we have an independent grasp of consistency, we have justified beliefs which are true. Ballaguer argues in favour of an independent sense of consistency of a (formal) system or construction: noticing consistency is taken as a faculty independent of an elucidation of consistency in model theory or logic (cf. Ballaguer 1998, pp.48-75).

Now, consistency can, of course, not be required of paraconsistent constructions or formal systems. These system and constructions, however, employ non-triviality as their substitute for consistency. A system has to be 
shown to be non-trivial, even if inconsistent. One can thus endorse the position of Hyper-Plentitudenous Platonism: all non-trivial abstract realms exist. This Inconsistent Platonism inherits all the virtues of consistent Platonism (i.e. the arguments concerning the applicability of mathematics etc. can be used here as well). One has to claim that we possess an independent faculty of discerning non-trivial but nonetheless coherent (i.e. systematic and explanatory) formal constructions and systems. I don't think that this is much more than is demanded by Plentitudenous Platonism. The whole development of paraconsistent logics in the last 35 years bears witness to this. In fact one may assume that we even more easily grasp nontriviality than consistency. Therefore Inconsistent Platonism or HyperPlentitudenous Platonism is an option!

The theory fits paraconsistent set theories and circumvents the troubles noneism runs into. Object talk and quantification are understood as always. They only - but obviously tremendous - difference to the common understanding of sets as abstract entities is the thesis that there exist many, many inconsistent abstract entities.

Inconsistent ontology is hard to swallow when talking about objects in space and time: How can it be that there is at some location in space time some atomic structure and yet another or the absence of the first? For abstract entities this is different: possessing two inconsistent properties means possessing two properties so that one of them implies that a sentence negating the possession of the other is true of the object in question. This makes a contradiction true of the objects in questions. Dialetheists endorse such contradictions. They argue that having a proof for each side of the contradiction no more can be required in standards of justified belief. As abstract entities are not in space and time - or at some other place - the incomprehensibility of possessing two contrary properties simply does not apply. Often we are endowed with criteria for attributing $F$ and another set of criteria for attributing non- $F$. If these independent routes of justifying a property ascription lead to a contradiction, then so be it for the Dialetheist.

Inconsistent Platonism in addition to meeting (O2) like the Platonist and inheriting some general arguments for Platonism meeting (O1) brings with it further resources in meeting $(\mathrm{O} 1)$ :

- all those intuitions in favour of the existence of $U$ (i.e. that our concept of SET entails the existence of a collected unity of sets),

- all those against a set/class-distinctions (i.e. that we have one basic concept ABSTRACT COLLECTION, which is not divided into SET and CLASS, a distinction introduced only be the needs of some theories in mathematics), 
- all those against the incomprehensible idea of an incompletable universe (i.e. in favour of the thesis that QUANTIFICATION is tied to a Domain Principle),

- all those against special objects we can talk about, but at the same time have to exclude from a broader domain of quantification (i.e. against a liberal use of axiomatic ontology to ensure a special status for $\mathrm{V})$.

Thus Inconsistent Platonism is a live option, it seems. The main difficulties with Inconsistent Platonism are also obvious: One may not like abstract entities in the first place, and one may not like inconsistent ontologies at all.

If an inconsistent or noneist ontology is too much to swallow when taking on such a paraconsistent system, then we have to opt for at least partial fictionalism with respect to (some) entities proposed within paraconsistent set theories. Then the exploration of universality in set theory naturally awaits a further thorough exploration of fictionalism. Too many difficult questions wait there: Fictions like fictional characters in literature depend historically and genetically on their authors, and maybe on still existent copies of the literary work and living readers (cf. Thomasson 1999), nothing of this sort can be said of pure sets. Pure sets (like in ZFC) are not just presented as abstract entities outside of space and time, but their presentation (the story told by $\mathbf{Z F C}$ ) arguably does not depend on any particular set theorist - not even Cantor, Frege or Zermelo. There might be several intermediate ontological categories between such purely abstract entities and spatio-temporal entities (cf. Thomasson 1999, pp. 120-33). Even in ZFCU one may wonder about the singletons of contingent urelements like the Cologne Cathedral: It seems bizarre to assume it to exist before the building was finished or even planned, thus this set seems to have a historical place! The recent interest in fictionalism may lead to increased ontological options - but don't hold your breath! 